\title{
A Representation of L-domain by Formal Concept Analysis
}

Wang shengwen ( $\sim$ wangshw7201@163.com )

Liupanshui Normal University https://orcid.org/0000-0002-2744-3085

Qingguo Li

HNU: Hunan University

\section{Research Article}

Keywords: LDF-context, G-connection, L-domain, Scott-continuous function, Categorical equivalence, Formal concept analysis

Posted Date: January 24th, 2022

DOI: https://doi.org/10.21203/rs.3.rs-1081137/v1

License: (c) (1) This work is licensed under a Creative Commons Attribution 4.0 International License. Read Full License 


\title{
A Representation of L-domain by Formal Concept Analysis
}

\author{
Shengwen Wang ${ }^{1,2^{*}}$ and Qingguo $\mathrm{Li}^{1 \dagger}$
}

${ }^{1}$ School of Mathematics, Hunan University, Lushan South Road, Changsha, 410082, Hunan, China.

${ }^{2}$ School of Mathematics and Computer Science, Liupanshui Normal University, Minghu Road, LiuPanshui, 553004, Guizhou, China.

*Corresponding author(s). E-mail(s): wangshw7201@163.com; Contributing authors: liqingguoli@aliyun.com; $\dagger$ These authors contributed equally to this work.

\begin{abstract}
In this paper, we represent L-domains by means of Formal Concept Analysis. Based on the attributive continuous formal contexts, we propose the notions of LDF-contexts and G-locally connections, and show that they provide concrete representations of L-domains and Scott-continuous functions between them, respectively. Moreover, the category of LDFcontexts with morphisms G-locally connections is proven to be equivalent to that of L-domains with morphisms Scott-continuous functions.
\end{abstract}

Keywords: LDF-context, G-connection, L-domain, Scott-continuous function, Categorical equivalence, Formal concept analysis

MSC Classification: 06B30, 06B35, 06F30, 18B30, 18B35

\section{Introduction}

Domain theory was introduced by Dana Scott [21] in the late of 1960s as a branch of order theory, for a purpose of modelling functional programming languages. Since then, different domain structures have been proposed and used as semantic domains for different programming languages [1, 22-24]. 
Formal Concept Analysis(FCA) was introduced by Rudolf Wille[3] as a mathematical theory for the formalization of concepts and conceptual thinking. It fiinds many applications in different disciplines such as Fuzzy Set Theory, Three-way Cognitive Analysis and Knowledge Processing, etc [6, 14, 16, 17, 20, 26]. Among others, an important application of FCA is to restructure complete lattices $[2,3]$.

In 2006, Zhang et al. [27] proposed the notion of approximable concepts and related domain theory to FCA by proving that every algebraic lattice arises as the lattice of approximable concepts of some formal context. Meanwhile, Hitzler et al. [10] constructed a suitable category based on the notion of approximable concepts, and showed that their category is equivalent to that of algebraic lattices and Scott-continuous functions. Following these two work, Lei and Luo [15] considered this correspondence in Rough Set Theory and proved that any algebraic lattice is isomorphic to some rough approximable concept lattice. Huang et al. [11] built the notion of $F$-approximable concepts and showed that $F$-approximable concepts and algebraic domains, rather than algebraic lattice, can interpret each other. Guo et al. [7] provided a representation of algebraic domains by using two variations of rough approximable concepts. Subsequently, similar structures were introduced to represent various algebraic domains, such as algebraic lattices in [18] and algebraic L-domains in [8].

All aforementioned representations are for subclasses of algebraic domains. Naturally, one wonders if one can characterize continuous domains in terms of FCA. To the best of our knowledge, an intimate relationship between general continuous domains and FCA were explicated by Guo et al. [9] and Wang et al. [25]. Generalizing techniques from classical formal concepts, in [25], they presented notions of continuous formal attribute concepts and attribute continuous formal contexts. By selecting a family of nonempty finite subsets of attributes in a formal context, they are able to show that every continuous domain can be generated by attribute continuous formal concepts.

The category LDOM of L-domains and Scott-continuous functions is a maximal Cartesian closed full subcategory of continuous domains, and is of its own interest $[12,13]$. The purpose of this paper is to characterize L-domains based on continuous formal attribute concepts. By defining a notion of $F$ sup in an attributive continuous formal context, we identify a special subclass of attributive continuous formal contexts, which we call an LDF-context. We show that the set of continuous formal concepts of an LDF-context forms an L-domains and each L-domain can be obtained in this way up to isomorphism. Moreover, we define the notion of G-locally connections between LDF-contexts and show that the category of L-domains and Scott-continuous functions is equivalent to that of LDF-contexts and G-locally connections.

The paper is organized as follows: Section 2 serves as a place to display some necessary preliminaries of domain theory and FCA and a representation theorem of continuous domains by means of continuous formal concepts. 
In Section 3, we present the notion of LDF-contexts and obtain a representation theorem of L-domains. In Section 4, we propose a new type of morphisms between LDF-contexts, called G-locally connections, and show there is a oneto-one correspondence between G-locally connections and Scott-continuous functions between L-domains. With all these ingredients, we establish the categorical equivalence between L-domains and LDF-contexts in Section 5.

\section{Preliminaries}

\subsection{Domain theory}

We recall some notions and results in domain theory $[2,4,5]$.

A poset $(P, \leq)$ with a least element is called pointed. For any subset $A$ of $P$, the down set $\downarrow A=\{x \in P \mid \exists a \in A, x \leq a\}$, and $\downarrow x=\downarrow\{x\}$. A subset $D$ of $P$ is directed if it is nonempty and every pair of elements in $D$ has an upper bound in $D$. A dcpo $P$ is a poset in which each directed subset $D$ of $P$ has a least upper bound $\bigvee D$ in $P$. A poset $P$ is called a complete lattice if every subset of $P$ has a least upper bound.

Let $P$ be a dcpo. We say that $x$ is way-below $y$, in symbols $x \ll y$, if and only if for any directed subset $D$ of $P$, the relation $y \leq \bigvee D$ always implies that $x \leq d$ for some $d \in D$. For any $X \subseteq P, \downarrow X=\{y \in P \mid(\exists x \in X) y \ll x\}$. And $\downarrow x=\downarrow\{x\}$. A basis $B_{P}$ of $P$ is a subset of $P$ in case the collection $B_{P} \cap \downarrow x$ is directed for $x \in P$ and has $x$ as a least upper bound.

Definition 1 A domain $P$ is a dcpo with a basis, and an $L$-domain $P$ is a domain with $\downarrow x$ being a complete lattice for each $x \in P$.

In a domain, the way-below relation satisfies the following interpolation property:

Proposition 1 Let $P$ be a domain, $M$ is a finite subset of $P$ with $M \ll y$. Then there exists $z \in P$ with $M \ll z \ll y$, where $M \ll y$ means that $x \ll y$ for all elements $x \in M$.

Definition 2 A Scott-continuous function is a function $f: P \rightarrow Q$ between two dcpos with $f(\bigvee D)=\bigvee_{x \in D} f(x)$, for all directed subset $D \subseteq P$.

\subsection{Formal concept analysis}

In this section, we highly recommend [3] as an introduction to classical FCA.

A formal context is a triple $\left(P_{o}, P_{a}, \models\right)$ where $P_{o}$ is a set of objects and $P_{a}$ is a set of attributes. The relation $\vDash$ is a subset of $P_{o} \times P_{a}$. In this case, two functions can be defined:

$$
\alpha: \mathcal{P}\left(P_{o}\right) \rightarrow \mathcal{P}\left(P_{a}\right), A \mapsto\left\{n \in P_{a} \mid \forall m \in A, m \vDash n\right\}
$$




$$
\omega: \mathcal{P}\left(P_{a}\right) \rightarrow \mathcal{P}\left(P_{o}\right), B \mapsto\left\{m \in P_{o} \mid \forall n \in B, m \vDash n\right\} .
$$

A formal (attribute) concept of a formal context $\left(P_{o}, P_{a}, \vDash\right)$ is a subset $B \subseteq P_{a}$ which is a fixed-point of $\alpha \circ \omega$.

For any set $X$, let $\mathcal{P}(X)$ and $\mathcal{F}(X)$ denote the powerset of $X$ and the family of all finite subsets of $X$, respectively. The notion $F \sqsubseteq X$ means that $F \in \mathcal{F}(X)$.

Definition 3 [25] Let $\mathbb{P}=\left(P_{o}, P_{a}, \vDash\right)$ be a formal context. A contractive operator on $\mathbb{P}$ is a mapping $\tau: \mathcal{P}\left(P_{a}\right) \rightarrow \mathcal{P}\left(P_{a}\right)$ which fulfils the following conditions, for any $B, C \subseteq P_{a}$,

1. (A1) $\tau(\alpha(\omega(B))) \subseteq \alpha(\omega(B))$;

2. (A2) $\tau(\tau(\alpha(\omega(B))))=\tau(\alpha(\omega(B)))$;

3. (A3) $\tau(\alpha(\omega(B))) \subseteq \tau(\alpha(\omega(C)))$ whenever $B \subseteq C$.

For convenience, we use $\lceil\bullet\rceil$ to denote $\tau(\alpha(\omega(\bullet)))$.

Definition 4 [25] Let $\left(P_{o}, P_{a}, \vDash\right)$ be a formal context and $\tau$ a contractive operator on $\left(P_{o}, P_{a}, \models\right)$. A $\tau$-consistent selection is a nonempty family $\mathcal{F}$ of nonempty finite subsets of $P_{a}$ which satisfies the following condition,

$(\mathbf{C A 1})(\forall F \in \mathcal{F}) M \sqsubseteq\lceil F\rceil \Rightarrow(\exists G \in \mathcal{F})(M \subseteq\lceil G\rceil, G \subseteq\lceil F\rceil)$.

In the sequels, we denote a $\tau$-consistent selection $\mathcal{F}$ by $\mathcal{F}_{\tau}$. For any $F \in \mathcal{F}_{\tau}$, since $F \neq \emptyset$, by condition (CA1), it is clear that $\lceil F\rceil \neq \emptyset$ and $\{\lceil G\rceil \mid G \in$ $\left.\mathcal{F}_{\tau}, G \subseteq\lceil F\rceil\right\} \neq \emptyset$.

Definition 5 [25] A structure $\mathbb{P}_{\tau}=\left(P_{o}, P_{a}, \vDash, \mathcal{F}_{\tau}\right)$ is said to be an attribute continuous formal context if $\left(P_{o}, P_{a}, \models\right)$ is a formal context and $\mathcal{F}_{\tau}$ is a $\tau$-consistent selection.

Definition 6 [25] Let $\mathbb{P}_{\tau}=\left(P_{o}, P_{a}, \vDash, \mathcal{F}_{\tau}\right)$ be an attribute continuous formal context. A continuous formal (attribute) concept of $\mathbb{P}_{\tau}$ is a subset $Q$ of $P_{a}$ which satisfies the following condition,

(CA2) $M \sqsubseteq Q \Rightarrow\left(\exists F \in \mathcal{F}_{\tau}\right) M \subseteq\lceil F\rceil \subseteq Q$.

$\mathfrak{B}\left(P_{a}, \mathcal{F}_{\tau}\right)$ denotes the set of all the continuous formal concepts. Taking $M=\emptyset$, condition (CA2) yields that every continuous formal concept $Q$ of $\mathbb{P}_{\tau}$ is not empty.

\subsection{Representation of domains}

In this section, we are to recall a systematic connection between domains and FCA and present a representation of domains in term of FCA. Let us review some continuous formal concepts of $\mathbb{P}_{\tau}$. 
Proposition 2 [25] Let $\mathbb{P}_{\tau}=\left(P_{o}, P_{a}, \vDash, \mathcal{F}_{\tau}\right)$ be an attribute continuous formal context. Then $\lceil F\rceil$ is a continuous formal concept of $\mathbb{P}_{\tau}$ for any $F \in \mathcal{F}_{\tau}$.

Proposition 3 [25] Let $Q$ be a continuous formal concept of an attribute continuous formal context $\mathbb{P}_{\tau}$. Then for any $M \sqsubseteq Q$,

1. $\lceil M\rceil \subseteq Q$.

2. There exists some $F \in \mathcal{F}_{\tau}$ such that $F \subseteq Q$ and $M \subseteq\lceil F\rceil \subseteq Q$.

Proposition 4 [25] Let $\mathbb{P}_{\tau}=\left(P_{o}, P_{a}, \vDash, \mathcal{F}_{\tau}\right)$ be an attribute continuous formal context and $Q$ a subset of $P_{a}$. Then the following are equivalent:

1. $Q$ is a continuous formal concept of $\mathbb{P}_{\tau}$.

2. The set $\left\{\lceil F\rceil \mid F \in \mathcal{F}_{\tau}, F \subseteq Q\right\}$ is directed and $Q=\bigcup\left\{\lceil F\rceil \mid F \in \mathcal{F}_{\tau}, F \subseteq\right.$ $Q\}$.

Lemma 5 [25] Let $\mathbb{P}_{\tau}=\left(P_{o}, P_{a}, \vDash, \mathcal{F}_{\tau}\right)$ be an attribute continuous formal context.

1. For any directed subset $\left\{Q_{i} \mid i \in I\right\}$ of $\mathfrak{B}\left(P_{a}, \mathcal{F}_{\tau}\right)$, the least upper bound $\bigvee_{i \in I} Q_{i}$ exists in $\mathfrak{B}\left(P_{a}, \mathcal{F}_{\tau}\right)$, and $\bigvee_{i \in I} Q_{i}=\bigcup_{i \in I} Q_{i}$.

2. For all continuous formal concepts $Q_{1}$ and $Q_{2}$ of $\mathbb{P}_{\tau}$, we have

$$
Q_{1} \ll Q_{2} \Leftrightarrow\left(\exists F \in \mathcal{F}_{\tau}\right)\left(Q_{1} \subseteq\lceil F\rceil, F \subseteq Q_{2}\right) .
$$

3. For any $F \in \mathcal{F}_{\tau}$,

$$
\lceil F\rceil \ll\lceil F\rceil \Leftrightarrow\left(\exists G \in \mathcal{F}_{\tau}\right)(\lceil F\rceil=\lceil G\rceil, G \subseteq\lceil G\rceil) .
$$

Theorem 6 (Representation theorem for domains) [25] Let $\mathbb{P}_{\tau}=\left(P_{o}, P_{a}, \vDash, \mathcal{F}_{\tau}\right)$ be an attribute continuous formal context. Then $\mathfrak{B}\left(P_{a}, \mathcal{F}_{\tau}\right)$ ordered by set inclusion forms a continuous domain.

Conversely, for every continuous domain $(D, \leq)$, there exists some attribute continuous formal context $\mathbb{P}_{\tau}$ such that $(D, \leq)$ is order isomorphic to $\left(\mathfrak{B}\left(P_{a}, \mathcal{F}_{\tau}\right), \subseteq\right)$.

\section{Representations of L-domains}

In this section, let us go further to discuss an attributive continuous formal context $\mathbb{P}_{\tau}=\left(P_{o}, P_{a}, \vDash, \mathcal{F}_{\tau}\right)$.

Definition 7 An attribute continuous formal context $\mathbb{P}_{\tau}=\left(P_{o}, P_{a}, \vDash, \mathcal{F}_{\tau}\right)$ is fitting if, for all $F \in \mathcal{F}_{\tau}$ and $A \sqsubseteq\lceil F\rceil$, there exists a set $F^{\prime} \in \mathcal{F}_{\tau}$ such that the following conditions hold:

1. (F1) $\lceil A\rceil \subseteq\left\lceil F^{\prime}\right\rceil \& F^{\prime} \subseteq\lceil F\rceil$,

2. $(\mathbf{F 2})\left(F^{\prime \prime} \in \mathcal{F}_{\tau}\right),\lceil A\rceil \subseteq\left\lceil F^{\prime \prime}\right\rceil \subseteq\lceil F\rceil \Rightarrow\left\lceil F^{\prime}\right\rceil \subseteq\left\lceil F^{\prime \prime}\right\rceil$.

In this case, $F^{\prime}$ is called an $F$-sup of $A$, and $\Omega(F, A)$ denotes the set of all F-sup of $A$. 
We aim to show that each fitting attributive continuous formal context denoted by LDF-context can generate an L-domain. To do this, let us appreciate a property about $\Omega(F, A)$.

Proposition 7 Let $\mathbb{P}_{\tau}=\left(P_{o}, P_{a}, \vDash, \mathcal{F}_{\tau}\right)$ be an LDF-context, $Q \in \mathfrak{B}\left(P_{a}, \mathcal{F}_{\tau}\right)$ and $F_{1}, F_{2} \in \mathcal{F}_{\tau}$. If $F_{1}, F_{2} \subseteq Q$ and $A_{1} \sqsubseteq A_{2} \sqsubseteq\left\lceil F_{1}\right\rceil \cap\left\lceil F_{2}\right\rceil$, then $\left\lceil F_{1}^{\prime}\right\rceil \subseteq\left\lceil F_{2}^{\prime}\right\rceil$ for all $F_{1}^{\prime} \in \Omega\left(F_{1}, A_{1}\right)$ and $F_{2}^{\prime} \in \Omega\left(F_{2}, A_{2}\right)$.

Proof Since $Q$ is an attribute continuous formal concept and $F_{1}, F_{2} \subseteq Q$, we have $F_{1} \cup F_{2} \sqsubseteq Q$. By condition (CA2), there is some $F \in \mathcal{F}_{\tau}$ such that $F_{1} \cup F_{2} \subseteq\lceil F\rceil \subseteq Q$, that is $F_{1} \subseteq\lceil F\rceil$ and $F_{2} \subseteq\lceil F\rceil$.

Let $M \in \Omega\left(F_{1}, A_{2}\right), N \in \Omega\left(F, A_{2}\right)$, we claim that $\lceil M\rceil=\lceil N\rceil$. By (F1) of Definition $7, M \in \Omega\left(F_{1}, A_{2}\right)$ implies $\left\lceil A_{2}\right\rceil \subseteq\lceil M\rceil$ and $M \subseteq\left\lceil F_{1}\right\rceil \subseteq\lceil F\rceil$, that is $\left\lceil A_{2}\right\rceil \subseteq\lceil M\rceil \subseteq\lceil F\rceil$. Thus, by (F2) of Definition 7, $\lceil N\rceil \subseteq\lceil M\rceil$. By (F1) of Definition $7, N \in \Omega\left(F, A_{2}\right)$ implies $\left\lceil A_{2}\right\rceil \subseteq\lceil N\rceil$ and $N \subseteq\lceil F\rceil$. As $\lceil N\rceil \subseteq\lceil M\rceil$ and $\lceil M\rceil \subseteq\left\lceil F_{1}\right\rceil$, we have $\left\lceil A_{2}\right\rceil \subseteq\lceil N\rceil \subseteq\lceil M\rceil \subseteq\left\lceil F_{1}\right\rceil$. It fellows that $\lceil M\rceil \subseteq\lceil N\rceil$ by (F2) of Definition 7. This implies that $\lceil M\rceil=\lceil N\rceil$.

Similarly, $\left\lceil F_{2}^{\prime}\right\rceil=\lceil N\rceil$ for $F_{2}^{\prime} \in \Omega\left(F_{2}, A_{2}\right)$ and $N \in \Omega\left(F, A_{2}\right)$. Thus $\lceil M\rceil=\left\lceil F_{2}^{\prime}\right\rceil$.

Since $M \in \Omega\left(F_{1}, A_{2}\right)$, by (F1) of Definition $7,\left\lceil A_{2}\right\rceil \subseteq\lceil M\rceil, M \subseteq\left\lceil F_{1}\right\rceil$. Because $A_{1} \subseteq A_{2}$, it is clear that $\left\lceil A_{1}\right\rceil \subseteq\lceil M\rceil \subseteq\left\lceil F_{1}\right\rceil$. Since $F_{1}^{\prime} \in \Omega\left(F_{1}, A_{1}\right)$, we obtain $\left\lceil F_{1}^{\prime}\right\rceil \subseteq\lceil M\rceil$ by $(\mathrm{F} 2)$ of Definition 7 , that is $\left\lceil F_{1}^{\prime}\right\rceil \subseteq\left\lceil F_{2}^{\prime}\right\rceil$.

Proposition 8 Let $\mathbb{P}_{\tau}=\left(P_{o}, P_{a}, \vDash, \mathcal{F}_{\tau}\right)$ be an LDF-context, $Q_{1}, Q_{2}, Q \in \mathfrak{B}\left(P_{a}, \mathcal{F}_{\tau}\right)$ and assume that $Q_{1} \cup Q_{2} \subseteq Q$. Set $\Im=\left\{K \mid\left(\exists M \sqsubseteq Q_{1} \cup Q_{2}\right)(\exists F \sqsubseteq Q) K \in \Omega(F, M)\right\}$ and $S=\bigcup_{K \in \Im}\lceil K\rceil$. Then $S$ is the least upper bound of $Q_{1}$ and $Q_{2}$ in $\downarrow Q$.

Proof First, we claim $Q_{1} \cup Q_{2} \subseteq S \subseteq Q$. Suppose $x \in Q_{1} \cup Q_{2}$. Then $x \in Q_{1}$ or $x \in Q_{2}$. If $x \in Q_{1}$, by Proposition 3, there is some $M_{x} \in \mathcal{F}_{\tau}$ such that $x \in$ $\left\lceil M_{x}\right\rceil \subseteq Q_{1} \subseteq Q$ and $M_{x} \subseteq Q_{1} \subseteq Q$. As $Q \in \mathfrak{B}\left(P_{a}, \mathcal{F}_{\tau}\right)$, there exists $F_{x} \in \mathcal{F}_{\tau}$ such that $M_{x} \subseteq\left\lceil F_{x}\right\rceil \subseteq Q$ and $F_{x} \subseteq Q$. Hence $K \in \Im$ for any $K \in \Omega\left(F_{x}, M_{x}\right)$. Since $x \in\left\lceil M_{x}\right\rceil \subseteq K$ and $K \subseteq\left\lceil F_{x}\right\rceil, x \in S$, this implies that $Q_{1} \subseteq S$. Similarly, $Q_{2} \subseteq S$. Thus $Q_{1} \cup Q_{2} \subseteq S$. Since for any $K \in \Im$, we know that there exists $M_{K} \sqsubseteq Q_{1} \cup Q_{2}$ and $F_{K} \sqsubseteq Q$ such that $K \in \Omega\left(F_{K}, M_{K}\right)$. By Definition $7, K \subseteq\left\lceil F_{K}\right\rceil$, that is $\lceil K\rceil \subseteq\left\lceil F_{K}\right\rceil \subseteq Q$. This implies that $S \subseteq Q$.

Second, we claim that $S \in \mathfrak{B}\left(P_{a}, \overline{\mathcal{F}}_{\tau}\right)$. By Proposition $2,\lceil K\rceil \in \mathfrak{B}\left(P_{a}, \mathcal{F}_{\tau}\right)$ for any $K \in \Im$. Let $K_{1}, K_{2} \in \Im$, there are $M_{i} \sqsubseteq Q_{1} \cup Q_{2}$ and $F_{i} \subseteq Q$ such that $K_{i} \in \Omega\left(F_{i}, M_{i}\right), i=1$, Since $M_{1}, M_{2} \sqsubseteq Q_{1} \cup Q_{2} \subseteq Q$, there is some $F_{3} \in \mathcal{F}_{\tau}$ such that $M_{1} \cup M_{2} \sqsubseteq\left\lceil F_{3}\right\rceil$ and $F_{3} \subseteq Q$. Taking $K_{3} \in \Omega\left(F_{3}, M_{1} \cup M_{2}\right)$, it is clear that $K_{3} \in \Im$. As $M_{1}, M_{2} \subseteq M_{1} \cup M_{2} \subseteq\left\lceil F_{3}\right\rceil$, by Proposition $7,\left\lceil K_{1}\right\rceil \subseteq\left\lceil K_{3}\right\rceil$ and $\left\lceil K_{2}\right\rceil \subseteq\left\lceil K_{3}\right\rceil$. This implies that $\{\lceil K\rceil \mid K \in \Im\}$ is directed. By Proposition 4, $S \in \mathfrak{B}\left(P_{a}, \mathcal{F}_{\tau}\right)$.

Finally, we claim that $S$ is the least upper bound of $Q_{1}$ and $Q_{2}$ in $\downarrow Q$. Suppose $Q_{3}$ is any upper bound of $Q_{1}$ and $Q_{2}$ in $\downarrow Q$. Let $K \in \Im$. Then there exists $M_{K} \sqsubseteq Q_{1} \cup Q_{2}$ and $F_{K} \subseteq Q$ such that $K \in \Omega\left(F_{K}, M_{K}\right)$. This implies that $M_{K} \subseteq Q_{3}$. So by Proposition 3, there is some $F \in \mathcal{F}_{\tau}$ such that $M_{K} \sqsubseteq\lceil F\rceil$ and $F \subseteq Q_{3}$, moreover, $\left\lceil M_{K}\right\rceil \subseteq\lceil F\rceil \subseteq Q_{3}$. Since $F_{K}, F \subseteq Q$, by the proof of Proposition $7,\lceil K\rceil=\left\lceil K_{1}\right\rceil$ for 
any $K_{1} \in \Omega\left(F, M_{K}\right)$. Therefore, $\lceil K\rceil \subseteq\lceil F\rceil \subseteq Q_{3}$, and hence $S \subseteq Q_{3}$. This implies that $S$ is the least upper bound of $Q_{1}$ and $Q_{2}$ in $\downarrow Q$.

Theorem 9 Let $\mathbb{P}_{\tau}=\left(P_{o}, P_{a}, \vDash, \mathcal{F}_{\tau}\right)$ be an LDF-context. Then $\left(\mathfrak{B}\left(P_{a}, \mathcal{F}_{\tau}\right), \subseteq\right)$ is an L-domain.

Proof Theorem 6 has shown that $\left(\mathfrak{B}\left(P_{a}, \mathcal{F}_{\tau}\right), \subseteq\right)$ is a continuous domain. To finish the proof, it is sufficient to check that for any element $Q$ of $\mathfrak{B}\left(P_{a}, \mathcal{F}_{\tau}\right)$, the set

$$
\downarrow Q=\left\{U \in \mathfrak{B}\left(P_{a}, \mathcal{F}_{\tau}\right) \mid U \subseteq Q\right\}
$$

is a complete lattice ordered by set inclusion.

By Proposition 8, we have that the least upper bound exists for every finite subset in $\downarrow Q$. Let $\left\{U_{i} \mid i \in I\right\}$ be a directed subset of $\downarrow Q$, it is obvious that $\bigcup_{i \in I} U_{i} \in \mathfrak{B}\left(P_{a}, \mathcal{F}_{\tau}\right)$, that is $\bigcup_{i \in I} U_{i} \in \downarrow Q$. This implies that $\downarrow Q$ is closed under sups of directed subsets.

The remainder is to prove that $\downarrow Q$ has a least element. Since $Q \in \mathfrak{B}\left(P_{a}, \mathcal{F}_{\tau}\right)$ and $\emptyset \sqsubseteq Q$, there is some $F_{\emptyset} \in \mathcal{F}_{\tau}$ such that $\emptyset \subseteq\left\lceil F_{\emptyset}\right\rceil \subseteq Q$ and $\Omega\left(F_{\emptyset}, \emptyset\right) \neq \emptyset$. Since $\emptyset \sqsubseteq U$ for any $U \in \downarrow Q$, there is some $F \in \mathcal{F}_{\tau}$ such that $F \subseteq U$ and $\emptyset \subseteq\lceil F\rceil$. Let $G_{\emptyset} \in \Omega\left(F_{\emptyset}, \emptyset\right)$, as $\emptyset \subseteq\lceil F\rceil$ and $F \subseteq Q$, it follows that $\left\lceil G_{\emptyset}\right\rceil \subseteq\lceil F\rceil$ by Proposition 7 , and hence $\left\lceil G_{\emptyset}\right\rceil \subseteq U$. This implies that $\left\lceil G_{\emptyset}\right\rceil$ is the least element of $\downarrow Q$.

Theorem 10 (Representation Theorem for L-domains) Let $(D, \leq)$ be an L-domain with a basis $B_{D}$. Then $\left(D, B_{D}, \geq, \mathcal{F}_{\tau}\right)$ denoted by $\mathcal{L}(D)$ is a LDF-context, and $(D, \leq)$ is order isomorphic to $\left(\mathfrak{B}\left(B_{D}, \mathcal{F}_{\tau}\right), \subseteq\right)$.

Proof By Theorem 6, $\mathcal{L}(D)$ is an attributive continuous formal context and $\left(\mathfrak{B}\left(B_{D}, \mathcal{F}_{\tau}\right), \subseteq\right)$ is isomorphic to $(D, \leq)$. Then the remainder is to prove that $\mathcal{L}(D)$ is fitting, that is $\Omega(F, M) \neq \emptyset$ for any $F \in \mathcal{F}_{\tau}$ and $M \sqsubseteq\lceil F\rceil$.

By the definition of $\mathcal{F}_{\tau}$, we have that $\bigvee F \in F \subseteq B_{D}$, it implies that $\lceil F\rceil=$ $\downarrow F \cap B_{D}=\downarrow \bigvee F \cap B_{D}$. Since $(D, \leq)$ is a continuous $L$-domain, $\downarrow \bigvee F$ is a complete lattice. Let $G=\left\{m_{F}\right\}$, where $m_{F}$ is the supremum of $M$ in $\downarrow \bigvee F$.

It is clear that $G \subseteq \downarrow \bigvee F \cap B_{D}$, by $M \subseteq \downarrow \bigvee F \cap B_{D}$. Thus,

$$
\lceil M\rceil=\downarrow M \cap B_{D} \subseteq \downarrow m_{F} \cap B_{D}=\lceil G\rceil
$$

Now assume that $G_{1} \in \mathcal{F}_{\tau}$ and $\lceil M\rceil \subseteq\left\lceil G_{1}\right\rceil \subseteq\lceil F\rceil$. Since $m_{F} \leq \bigvee G_{1} \leq \bigvee F$, it follows that $\downarrow m_{F} \cap B_{D} \subseteq \downarrow \bigvee G_{1} \cap B_{D}$. This implies that $\lceil G\rceil \subseteq\left\lceil G_{1}\right\rceil$.

By Definition 7, $G=\left\{m_{F}\right\}$ is an F-sup of $M$, that is, the set $\Omega(F, M)$ is not empty.

\section{4 category equivalence}

In this section, the category equivalence is established between the category of L-domains and that of LDF-contexts. Some basic notions and results about category theory can be referred to [19]. 


\subsection{G-locally connection}

Definition 8 Let $\mathbb{P}_{\tau}=\left(P_{o}, P_{a}, \models_{P}, \mathcal{F}_{\tau}\right)$ and $\mathbb{Q}_{\tau^{\prime}}=\left(Q_{o}, Q_{a}, \models_{Q}, \mathcal{F}_{\tau^{\prime}}\right)$ be LDFcontexts. A relation $\Theta \subseteq \mathcal{F}_{\tau} \times Q_{a}$ is called $a$ G-locally connection from $\mathbb{P}_{\tau}$ to $\mathbb{Q}_{\tau^{\prime}}$, written as $\Theta: \mathbb{P}_{\tau} \vdash \mathbb{Q}_{\tau^{\prime}}$, if for any $X, X_{1}, X_{2} \in \mathcal{F}_{\tau}$ and $G \sqsubseteq Q_{a}$,

1. $(\mathbf{G 1})\left(\exists y \in Q_{a}\right)(X, y) \in \Theta$.

2. (G2) $\left(X_{1} \sqsubseteq\left\lceil X_{2}\right\rceil \& X_{1} \Theta G\right) \Rightarrow X_{2} \Theta G \& X_{1} \Theta\lceil G\rceil$;

3. (G3) $X \Theta G \Rightarrow\left(\exists X^{\prime} \in \mathcal{F}_{\tau} \& G^{\prime} \in \mathcal{F}_{\tau^{\prime}}\right) X^{\prime} \subseteq\lceil X\rceil \& X^{\prime} \Theta G^{\prime} \& G \sqsubseteq\left\lceil G^{\prime}\right\rceil$.

where $X \Theta G$ means $(X, y) \in \Theta$ for any $y \in G$.

Proposition 11 Let $\Theta: \mathbb{P}_{\tau} \vdash \mathbb{Q}_{\tau^{\prime}}$ be a G-locally connection.

1. For any $X \in \mathcal{F}_{\tau}, \bigcup\left\{\lceil Y\rceil \mid Y \in \mathcal{F}_{\tau^{\prime}} \& X \Theta Y\right\}$ is a attributive continuous formal concept of $\mathbb{Q}_{\tau^{\prime}}$.

2. For any attributive continuous concept $F$ of $\mathbb{P}_{\tau}$, the set $\left\{y \in Q_{a} \mid(\exists X \in\right.$ $\left.\left.\mathcal{F}_{\tau}\right) X \sqsubseteq F \&(X, y) \in \Theta\right\}$ is an attributive continuous concept of $\mathbb{Q}_{\tau^{\prime}}$.

Proof (1) Suppose $M \sqsubseteq \bigcup\left\{\lceil Y\rceil \mid Y \in \mathcal{F}_{\tau^{\prime}} \& X \Theta Y\right\}$. Let $x \in M$, then there exists $Y_{x} \in \mathcal{F}_{\tau^{\prime}}$ satisfying $X \Theta Y_{x}$ such that $x \in\left\lceil Y_{x}\right\rceil$. Since $Y_{x} \subseteq \bigcup_{x \in M} Y_{x}$, so $\left\lceil Y_{x}\right\rceil \subseteq\left\lceil\bigcup_{x \in M} Y_{x}\right\rceil$, that is $\bigcup_{x \in M}\left\lceil Y_{x}\right\rceil \subseteq\left\lceil\bigcup_{x \in M} Y_{x}\right\rceil$. By Definition 8, $X \Theta Y_{x}$ implies $X \Theta \bigcup_{x \in M} Y_{x}$. Thus, $\bigcup_{x \in M} Y_{x} \in \mathcal{F}_{\tau^{\prime}}$ such that $M \subseteq\left\lceil\bigcup_{x \in M} Y_{x}\right\rceil \subseteq \bigcup\{\lceil Y\rceil \mid$ $\left.Y \in \mathcal{F}_{\tau^{\prime}} \& X \Theta Y\right\}$.

(2) Let $N=\left\{y \in Q_{a} \mid\left(\exists X \in \mathcal{F}_{\tau}\right) X \sqsubseteq F \&(X, y) \in \Theta\right\}$. For any $G \sqsubseteq N$ and $y \in G$, there exists $X_{y} \in \mathcal{F}_{\tau}$ such that $X_{y} \sqsubseteq F$ and $\left(X_{y}, y\right) \in \Theta$. Let $X_{G}=$ $\bigcup_{y \in G} X_{y} \sqsubseteq F$. Since $F$ is an attributive continuous concept of $\mathbb{P}_{\tau}$, there is $X \in \mathcal{F}_{\tau}$ such that $X_{G} \sqsubseteq\lceil X\rceil \subseteq F$. According to the condition (G2) of Definition 8, for any $y \in G,(X, y) \in \Theta$, that is $X \Theta G$. By the condition (G3) of Definition 8, there is $X^{\prime} \in \mathcal{F}_{\tau}$ and $G^{\prime} \in \mathcal{F}_{\tau^{\prime}}$ such that $X^{\prime} \sqsubseteq\lceil X\rceil$ and $X^{\prime} \Theta G^{\prime}$ and $G \sqsubseteq\left\lceil G^{\prime}\right\rceil$. Since $X^{\prime} \in \mathcal{F}_{\tau}$ and $X^{\prime} \sqsubseteq F$, so $\left\lceil G^{\prime}\right\rceil \subseteq N$. We have $G \sqsubseteq\left\lceil G^{\prime}\right\rceil \subseteq N$, this shows that $N$ is an attributive continuous concept of $\mathbb{Q}_{\tau^{\prime}}$.

The following theorem tells us that there is a one-to-one correspondence between Scott-continuous functions from $\left(\mathfrak{B}\left(P_{a}, \mathcal{F}_{\tau}\right), \subseteq\right)$ to $\left(\mathfrak{B}\left(Q_{a}, \mathcal{F}_{\tau^{\prime}}\right), \subseteq\right)$ and G-locally connections from $\mathbb{P}_{\tau}$ to $\mathbb{Q}_{\tau^{\prime}}$.

Theorem 12 Let $\mathbb{P}_{\tau}$ and $\mathbb{Q}_{\tau^{\prime}}$ be LDF-contexts.

1. For any G-locally connection $\Theta: \mathbb{P}_{\tau} \vdash \mathbb{Q}_{\tau^{\prime}}$, define a map $\phi_{\Theta}$ : $\mathfrak{B}\left(P_{a}, \mathcal{F}_{\tau}\right) \rightarrow \mathfrak{B}\left(Q_{a}, \mathcal{F}_{\tau^{\prime}}\right)$ by

$$
\phi_{\Theta}(F)=\left\{y \in Q_{a} \mid\left(\exists X \in \mathcal{F}_{\tau}\right) X \sqsubseteq F \&(X, y) \in \Theta\right\} .
$$

Then $\phi_{\Theta}$ is a Scott-continuous function. 
2. Conversely, for any Scott-continuous function $\phi: \mathfrak{B}\left(P_{a}, \mathcal{F}_{\tau}\right) \rightarrow$ $\mathfrak{B}\left(Q_{a}, \mathcal{F}_{\tau^{\prime}}\right)$, define a relation $\Theta_{\phi} \subseteq \mathcal{F}_{\tau} \times Q_{a}$ by

$$
(X, y) \in \Theta_{\phi} \Leftrightarrow y \in \phi(\lceil X\rceil)
$$

Then $\Theta_{\phi}$ is a G-locally connection.

3. Moreover, we have $\Theta=\Theta_{\phi_{\Theta}}$ and $\phi=\phi_{\Theta_{\phi}}$

Proof (1) It follows from Proposition 11 that $\phi_{\Theta}$ is well defined. It is obvious that $\phi_{\Theta}$ is order-preserving. Let $\left\{E_{i}\right\}_{i \in I}$ be a directed family of attributive continuous formal concepts of $\mathbb{P}_{\tau}$. Then the family $\left\{\phi_{\Theta}\left(E_{i}\right)\right\}_{i \in I}$ is a directed family of attributive continuous formal concepts of $\mathbb{Q}_{\tau^{\prime}}$. By Proposition $4, \bigvee_{i \in I} E_{i}=\bigcup_{i \in I} E_{i}$ and $\bigvee_{i \in I} \phi_{\Theta}\left(E_{i}\right)=\bigcup_{i \in I} \phi_{\Theta}\left(E_{i}\right)$. To end this proof, it is sufficient to check that $\phi_{\Theta}\left(\bigcup_{i \in I} E_{i}\right)=\bigcup_{i \in I} \phi_{\Theta}\left(E_{i}\right)$. It is clear that the inclusion $\bigcup_{i \in I} \phi_{\Theta}\left(E_{i}\right) \subseteq$ $\phi_{\Theta}\left(\bigcup_{i \in I} E_{i}\right)$ is true. Let $y \in \phi_{\Theta}\left(\bigcup_{i \in I} E_{i}\right)$, then there exists $X \in \mathcal{F}_{\tau}$ such that $X \sqsubseteq \bigcup_{i \in I} E_{i}$ and $(X, y) \in \Theta$. As $\left\{E_{i}\right\}_{i \in I}$ is directed, there exists $i_{0} \in I$ such that $X \sqsubseteq E_{i_{0}}$. This implies that $y \in \phi_{\Theta}\left(E_{i_{0}}\right)$ and thus $\phi_{\Theta}\left(\bigcup_{i \in I} E_{i}\right) \subseteq \bigcup_{i \in I} \phi_{\Theta}\left(E_{i}\right)$.

(2) Given a Scott-continuous function $\phi$ from $\mathfrak{B}\left(P_{a}, \mathcal{F}_{\tau}\right)$ to $\mathfrak{B}\left(Q_{a}, \mathcal{F}_{\tau^{\prime}}\right)$, it is obvious that $\Theta_{\phi}$ from $\mathbb{P}_{\tau}$ to $\mathbb{Q}_{\tau^{\prime}}$ satisfies condition (G1). We claim that $\Theta_{\phi}$ is a G-locally connection by checking that $\Theta_{\phi}$ satisfies conditions (G2) and (G3). Let $X_{1}, X_{2}, X \in \mathcal{F}_{\tau}$ and $G \sqsubseteq Q_{a}$.

For condition (G2), if $X_{1} \sqsubseteq\left\lceil X_{2}\right\rceil$ and $X_{1} \Theta_{\phi} G$, then $G \sqsubseteq \phi\left(\left\lceil X_{1}\right\rceil\right)$. Since $\left\lceil X_{1}\right\rceil \in$ $\mathfrak{B}\left(P_{a}, \mathcal{F}_{\tau}\right), \phi\left(\left\lceil X_{1}\right\rceil\right) \in \mathfrak{B}\left(Q_{a}, \mathcal{F}_{\tau^{\prime}}\right)$ by the part $(2)$ of proposition 11 . This implies $\lceil G\rceil \subseteq \phi\left(\left\lceil X_{1}\right\rceil\right)$, that is $X_{1} \Theta_{\phi}\lceil G\rceil$. Since $X_{1} \sqsubseteq\left\lceil X_{2}\right\rceil,\left\lceil X_{1}\right\rceil \subseteq\left\lceil X_{2}\right\rceil$, this implies $\phi\left(\left\lceil X_{1}\right\rceil\right) \subseteq \phi\left(\left\lceil X_{2}\right\rceil\right)$ by the Scott-continuous function $\phi$. Thus $G \subseteq \phi\left(\left\lceil X_{2}\right\rceil\right)$, that is $X_{2} \Theta_{\phi} G$.

For condition (G3), let $X \Theta_{\phi} G$, then $G \sqsubseteq \phi(\lceil X\rceil)$. Since $\lceil X\rceil \in \mathfrak{B}\left(P_{a}, \mathcal{F}_{\tau}\right),\lceil X\rceil$ is the directed union of the set of $\left\{\left\lceil X^{\prime}\right\rceil \mid X^{\prime} \in \mathcal{F}_{\tau}, X^{\prime} \sqsubseteq\lceil X\rceil\right\}$. Since $\phi$ is a Scott-continuous function, we have

$$
\phi(\lceil X\rceil)=\phi\left(\bigcup\left\{\left\lceil X^{\prime}\right\rceil \mid X^{\prime} \in \mathcal{F}_{\tau}, X^{\prime} \sqsubseteq\lceil X\rceil\right\}\right)=\bigcup\left\{\phi\left(\left\lceil X^{\prime}\right\rceil\right) \mid X^{\prime} \in \mathcal{F}_{\tau}, X^{\prime} \sqsubseteq\lceil X\rceil\right\}
$$

Thus $G \sqsubseteq \phi\left(\left\lceil X^{\prime}\right\rceil\right)$ for some $X^{\prime} \in \mathcal{F}_{\tau}$ with $X^{\prime} \subseteq\lceil X\rceil$. For $G \sqsubseteq \phi\left(\left\lceil X^{\prime}\right\rceil \in \mathfrak{B}\left(Q_{a}, \mathcal{F}_{\tau^{\prime}}\right)\right.$, there exist some $G^{\prime} \in \mathcal{F}_{\tau^{\prime}}$ satisfying $G^{\prime} \subseteq \phi\left(\left\lceil X^{\prime}\right\rceil\right)$ and $G \sqsubseteq\left\lceil G^{\prime}\right\rceil$. To sum up, there exist some $X^{\prime} \in \mathcal{F}_{\tau}$ and $G^{\prime} \in \mathcal{F}_{\tau^{\prime}}$ such that $X^{\prime} \subseteq\lceil X\rceil, X^{\prime} \bar{\Theta}_{\phi} G^{\prime}, G \subseteq\left\lceil G^{\prime}\right\rceil$.

(3) $\Theta=\Theta_{\phi_{\Theta}}$ and $\phi=\phi_{\Theta_{\phi}}$.

For any $X \in \mathcal{F}_{\tau}$ and $y \in Q_{a}$, we have

$$
\begin{aligned}
(X, y) \in \Theta_{\phi} & \Leftrightarrow y \in \phi_{\Theta}(\lceil X\rceil) \\
& \Leftrightarrow\left(\exists X^{\prime} \in \mathcal{F}_{\tau}\right) X^{\prime} \sqsubseteq\lceil X\rceil \&\left(X^{\prime}, y\right) \in \Theta \\
& \Leftrightarrow X \Theta\{y\} \\
& \Leftrightarrow(X, y) \in \Theta .
\end{aligned}
$$

This implies $\Theta=\Theta_{\phi_{\Theta}}$.

For any attributive continuous formal concept $E$ of $\mathbb{P}_{\tau}$, we have

$$
\begin{aligned}
\phi_{\Theta_{\phi}}(E) & =\left\{y \in Q_{a} \mid\left(\exists X \in \mathcal{F}_{\tau}\right) X \sqsubseteq E \&(X, y) \in \Theta_{\phi}\right\} \\
& =\left\{y \in Q_{a} \mid\left(\exists X \in \mathcal{F}_{\tau}\right) X \sqsubseteq E \& y \in \phi(\lceil X\rceil)\right\} \\
& =\bigcup\left\{\phi(\lceil X\rceil) \mid X \in \mathcal{F}_{\tau} \& X \sqsubseteq E\right\}
\end{aligned}
$$




$$
\begin{aligned}
& =\phi\left(\bigcup\left\{\lceil X\rceil \mid X \in \mathcal{F}_{\tau} \& X \sqsubseteq E\right\}\right) \\
& =\phi(E) .
\end{aligned}
$$

This implies $\phi=\phi_{\Theta_{\phi}}$.

Similar to the proof of Theorem 12, we have

Theorem 13 Let $\left(D_{1}, \leq_{1}\right)$ and $\left(D_{2}, \leq_{2}\right)$ are continuous L-domains.

1. For any Scott-continuous function $\Psi: D_{1} \rightarrow D_{2}$, define a relation $\Omega_{\psi} \subseteq$ $\mathcal{F}_{\tau_{1}} \times B_{D_{2}}$ by

$$
(F, c) \in \Omega_{\psi} \Leftrightarrow c \ll_{2} \psi(\bigvee F) .
$$

$F \subseteq B_{D_{1}}$ with $\bigvee F \in F$, Then $\Omega_{\psi}$ is a G-locally connection from $\mathcal{L}\left(D_{1}\right)$ to $\mathcal{L}\left(D_{2}\right)$.

2. Conversely, for any $G$-locally connection $\Omega: \mathcal{L}\left(D_{1}\right) \vdash \mathcal{L}\left(D_{2}\right)$, define a function $\psi_{\Omega}: D_{1} \rightarrow D_{2}$ by

$$
\psi_{\Omega}(x)=\bigvee\left\{c \in B_{D_{2}} \mid\left(\exists F \in \mathcal{F}_{\tau_{1}}\right) F \sqsubseteq \downarrow x \cap B_{D_{1}},(F, c) \in \Omega\right\}
$$

Then $\psi_{\Omega}$ is a Scott-continuous function.

3. Moreover, we have $\psi=\psi_{\Omega_{\psi}}$ and $\Omega=\Omega_{\psi_{\Omega}}$.

\subsection{Categorical equivalence}

First, we claim that LDF-contexts and G-locally connections do form a category. Given an LDF-context $\mathbb{P}_{\tau}=\left(P_{o}, P_{a}, \models_{P}, \mathcal{F}_{\tau}\right)$, define a relation $i d_{\mathbb{P}_{\tau}} \subseteq$ $\mathcal{F}_{\tau} \times P_{a}$ by

$$
(F, x) \in i d_{\mathbb{P}_{\tau}} \Leftrightarrow x \in\lceil F\rceil
$$

Let $\mathbb{P}_{\tau}, \mathbb{Q}_{\tau^{\prime}}$ and $\mathbb{S}_{\tau^{\prime \prime}}$ be LDF-contexts; let $\Theta: \mathbb{P}_{\tau} \vdash \mathbb{Q}_{\tau^{\prime}}$ and $\Upsilon: \mathbb{Q}_{\tau^{\prime}} \vdash \mathbb{S}_{\tau^{\prime \prime}}$ be G-locally connections. Define a relation $\Upsilon \circ \Theta \subseteq \mathcal{F}_{\tau} \times S_{a}$ by

$$
(X, z) \in(\Upsilon \circ \Theta) \Leftrightarrow\left(\exists Y \in \mathcal{F}_{\tau^{\prime}}\right) X \Theta Y \&(Y, z) \in \Upsilon
$$

It is trivial to check that $i d_{\mathbb{P}_{\tau}}$ is a G-locally connection from $\mathbb{P}_{\tau}$ to itself and $\Upsilon \circ \Theta$ is a G-locally connection from $\mathbb{P}_{\tau}$ to $\mathbb{S}_{\tau^{\prime \prime}}$.

Now, we use LDFC to denote the category of LDF-contexts with G-locally connections as morphisms, and LDOM to denote the category of L-domains with Scott-continuous functions.

Based on Theorem 9, for any LDF-context $\mathbb{P}_{\tau}$, define a map $\mathcal{G}_{0}: \mathbf{L D F C}_{0} \rightarrow$ $\mathbf{L D O M}_{0}$ by

$$
\mathcal{G}_{0}\left(\mathbb{P}_{\tau}\right)=\mathfrak{B}\left(P_{a}, \mathcal{F}_{\tau}\right)
$$

Based on Theorem 12, for any G-locally connection $\Theta$ from $\mathbb{P}_{\tau}$ to $\mathbb{Q}_{\tau^{\prime}}$, define a map $\mathcal{G}_{a}: \mathbf{L D F C}_{a} \rightarrow \mathbf{L D O M}$ by

$$
\mathcal{G}_{a}(\Theta)=\psi_{\Theta}
$$


That is, for any LDF-context $E$ of $\mathbb{P}_{\tau}$,

$$
\mathcal{G}_{a}(\Theta)(E)=\left\{y \in Q_{a} \mid\left(\exists X \in \mathcal{F}_{\tau}\right) X \sqsubseteq E \&(X, y) \in \Theta\right\}
$$

Proposition $14 \mathcal{G}=\left(\mathcal{G}_{0}, \mathcal{G}_{a}\right)$ is a functor from LDFC to LDOM.

Proof First, for any LDF-context $\mathbb{P}_{\tau}$, by Proposition 4, we have that $E=\bigcup\{\lceil X\rceil \mid$ $\left.X \in \mathcal{F}_{\tau} \& X \sqsubseteq E\right\}$ for any attributive continuous formal concept $E$ of $\mathbb{P}_{\tau}$, then $\mathcal{G}_{a}\left(i d_{\mathbb{P}_{\tau}}\right)$ is the same as $i d_{\mathcal{G}_{0}\left(\mathbb{P}_{\tau}\right)}$. Second, For LDF-contexts $\mathbb{P}_{\tau}, \mathbb{Q}_{\tau^{\prime}}$ and $\mathbb{S}_{\tau^{\prime \prime}}$, let $\Theta: \mathbb{P}_{\tau} \vdash \mathbb{Q}_{\tau^{\prime}}$ and $\Upsilon: \mathbb{Q}_{\tau^{\prime}} \vdash \mathbb{S}_{\tau^{\prime \prime}}$. For any attributive continuous formal concept $E$ of $\mathbb{P}_{\tau}$ and $z \in S_{a}$, we have

$$
\begin{aligned}
z \in \mathcal{G}_{a}(\Upsilon \circ \Theta)(E) & \Leftrightarrow\left(\exists X \in \mathcal{F}_{\tau}\right) X \sqsubseteq E \&(X, z) \in \Upsilon \circ \Theta \\
& \Leftrightarrow\left(\exists X \in \mathcal{F}_{\tau}, Y \in \mathcal{F}_{\tau^{\prime}}\right) X \sqsubseteq E \& X \Theta Y \&(Y, z) \in \Upsilon \\
& \Leftrightarrow\left(\exists Y \in \mathcal{F}_{\tau^{\prime}}\right) Y \sqsubseteq \mathcal{G}_{a}(\Theta)(E) \&(Y, z) \in \Upsilon \\
& \Leftrightarrow z \in \mathcal{G}_{a}(\Upsilon)\left(\mathcal{G}_{a}(\Theta)(E)\right)
\end{aligned}
$$

That is $\mathcal{G}_{a}(\Upsilon \circ \Theta)=\mathcal{G}_{a}(\Upsilon) \circ \mathcal{G}_{a}(\Theta)$.

Similarly, we will obtain a functor from LDOM to LDFC. For any Ldomain $(D, \leq)$, the notion $i d_{D}$ denotes the identity map on $(D, \leq)$

Based on Theorem 10, for any L-domain $(D, \leq)$ with basis $B_{D}$, define a map $\mathcal{H}_{0}: \mathbf{L D O M}_{0} \rightarrow \mathbf{L D F C}_{0}$ by

$$
\mathcal{H}_{0}(D)=\left(D, B_{D}, \geq, \mathcal{F}_{\tau}\right)\left(\text { i.e., } \mathcal{H}_{0}(D)=\mathcal{L}(D)\right)
$$

Based on Theorem 13, for any Scott-continuous function $\psi: D_{1} \rightarrow D_{2}$, define a map $\mathcal{H}_{a}: \mathbf{L D O M}_{a} \rightarrow \mathbf{L D F C}{ }_{a}$ by

$$
\mathcal{H}_{a}(\psi)=\Omega_{\psi}
$$

That is, for any $F \in \mathcal{F}_{\tau_{1}}$ and $c \in B_{D_{2}}$,

$$
(F, c) \in \mathcal{H}_{a}(\psi) \Leftrightarrow c \ll_{2} \psi(\bigvee F)
$$

Proposition $15 \mathcal{H}=\left(\mathcal{H}_{0}, \mathcal{H}_{a}\right)$ is a functor from LDOM to LDFC.

Proof For any $F \sqsubseteq B_{D}$ with $\bigvee F \in F$ and $c \in B_{D}$, we have

$$
\begin{aligned}
(F, c) \in \mathcal{H}_{a}\left(i d_{D}\right) & \Leftrightarrow c \ll i d_{D}(\bigvee F)=\bigvee F \\
& \Leftrightarrow c \in \downarrow \bigvee F \cap B_{D} \\
& \Leftrightarrow c \in\lceil F\rceil \\
& \Leftrightarrow(F, c) \in i d_{\mathcal{H}_{0}(D)}
\end{aligned}
$$

That is $\mathcal{H}_{a}\left(i d_{D}\right)=i d_{\mathcal{H}_{0}(D)}$. 
Suppose $\left(D_{1}, \leq_{1}\right),\left(D_{2}, \leq_{2}\right)$ and $\left(D_{3}, \leq_{3}\right)$ are L-domains. Let $\psi: D_{1} \rightarrow D_{2}$ and $\psi^{\prime}: D_{2} \rightarrow D_{3}$ be Scott-continuous functions. for any $F \in \mathcal{F}_{\tau_{1}}$ and $c \in B_{D_{3}}$, we have

$$
\begin{aligned}
(F, c) \in \mathcal{H}_{a}\left(\psi^{\prime} \circ \psi\right) & \Leftrightarrow c \ll_{3} \psi^{\prime}(\psi(\bigvee F)) \\
& \Leftrightarrow c \ll_{3} \bigvee \psi^{\prime}\left(\downarrow \psi(\bigvee F) \cap B_{D_{2}}\right) \\
& \Leftrightarrow\left(\exists c^{\prime} \in \downarrow \psi(\bigvee F) \cap B_{D_{2}}\right) c \ll_{3} \psi^{\prime}\left(c^{\prime}\right) \\
& \Leftrightarrow\left(\exists F^{\prime} \in \mathcal{F}_{\tau_{2}}\right) F \mathcal{H}_{a}(\psi) F^{\prime} \& F^{\prime} \mathcal{H}_{a}\left(\psi^{\prime}\right) c \\
& \Leftrightarrow(F, c) \in \mathcal{H}_{a}\left(\psi^{\prime}\right) \circ \mathcal{H}_{a}(\psi)
\end{aligned}
$$

That is $\mathcal{H}_{a}\left(\psi^{\prime} \circ \psi\right)=\mathcal{H}_{a}\left(\psi^{\prime}\right) \circ \mathcal{H}_{a}(\psi)$.

For any L-domain $(D, \leq)$, define a map $\psi_{D}: D \rightarrow\left(\mathfrak{B}\left(B_{D}, \mathcal{F}_{\tau}\right), \subseteq\right)$ by

$$
\psi_{D}(x)=\downarrow x \cap B_{D}
$$

From Theorem 6 and Theorem 10, $\psi_{D}$ is an isomorphism from $(D, \leq)$ to $\left(\mathfrak{B}\left(B_{D}, \mathcal{F}_{\tau}\right), \subseteq\right)$.

For any LDF-context $\mathbb{P}_{\tau}=\left(P_{0}, P_{a}, \models, \mathcal{F}_{\tau}\right)$, the notation $B_{\mathfrak{B}\left(P_{a}, \mathcal{F}_{\tau}\right)}$ is the basis of $\left(\mathfrak{B}\left(P_{a}, \mathcal{F}_{\tau}\right), \subseteq\right)$. From Lemma $5, B_{\mathfrak{B}\left(P_{a}, \mathcal{F}_{\tau}\right)}=\left\{\lceil X\rceil \mid X \in \mathcal{F}_{\tau}\right\}$. Define a relation $\Theta_{\mathbb{P}_{\tau}} \subseteq \mathcal{F}_{\tau} \times B_{\mathfrak{B}\left(P_{a}, \mathcal{F}_{\tau}\right)}$ by

$$
\left(X,\left\lceil X^{\prime}\right\rceil\right) \in \Theta_{\mathbb{P}_{\tau}} \Leftrightarrow X^{\prime} \subseteq\lceil X\rceil
$$

It is trivial to check that $\Theta_{\mathbb{P}_{\tau}}$ is a G-locally connection from $\mathbb{P}_{\tau}$ to $\mathcal{H}_{0}\left(\mathcal{G}_{0}\left(\mathbb{P}_{\tau}\right)\right)=$ $\left(\mathfrak{B}\left(P_{a}, \mathcal{F}_{\tau}\right), B_{\mathfrak{B}\left(P_{a}, \mathcal{F}_{\tau}\right)}, \supseteq, \mathcal{F}_{\tau *}\right)$.

In the sequels, define a relation $\Omega_{\mathbb{P}_{\tau}} \subseteq \mathcal{F}_{\tau *} \times P_{a}$ by

$$
(\alpha, x) \in \Omega_{\mathbb{P}_{\tau}} \Leftrightarrow x \in \bigcup \alpha
$$

Routine checks verify that $\Omega_{\mathbb{P}_{\tau}}$ is a G-locally connection from $\mathcal{H}_{0}\left(\mathcal{G}_{0}\left(\mathbb{P}_{\tau}\right)\right)$ to $\mathbb{P}_{\tau}$ and the inverse of $\Theta_{\mathbb{P}_{\tau}}$. That is, $\Theta_{\mathbb{P}_{\tau}}$ is an isomorphism in LDFC.

Proposition 16 Let $\left(D_{1}, \leq_{1}\right)$ and $\left(D_{2}, \leq_{2}\right)$ be continuous L-domains. For any Scott-continuous function $\psi: D_{1} \rightarrow D_{2}$,

$$
\psi_{D_{2}} \circ \psi=\mathcal{G}_{a}\left(\mathcal{H}_{a}(\psi)\right) \circ \psi_{D_{1}}
$$

Let $\mathbb{P}_{\tau}$ and $\mathbb{Q}_{\tau^{\prime}}$ be LDF-contexts. For any G-locally connection $\Theta: \mathbb{P}_{\tau} \vdash \mathbb{Q}_{\tau^{\prime}}$,

$$
\Theta_{\mathbb{Q}_{\tau^{\prime}}} \circ \Theta=\mathcal{H}_{a}\left(\mathcal{G}_{a}(\Theta)\right) \circ \Theta_{\mathbb{P}_{\tau}}
$$

Proof By the proposition of Scott-continuous functions, the first equation is true.

For any $X \in \mathcal{F}_{\tau}$ and $Y \in \mathcal{F}_{\tau^{\prime}}$, we have

$$
\begin{aligned}
(X,\lceil Y\rceil) \in \mathcal{H}_{a}\left(\mathcal{G}_{a}(\Theta)\right) \circ \Theta_{\mathbb{P}_{\tau}} & \Leftrightarrow\left(\exists \alpha \in \mathcal{F}_{\tau *}\right) X \Theta_{\mathbb{P}_{\tau}} \alpha \&(\alpha,\lceil Y\rceil) \in \mathcal{H}_{a}\left(\mathcal{G}_{a}(\Theta)\right) \\
& \Leftrightarrow\left(\exists X^{\prime} \in \mathcal{F}_{\tau}\right) X \Theta_{\mathbb{P}_{\tau}}\left\lceil X^{\prime}\right\rceil \&\lceil Y\rceil \subseteq \mathcal{G}_{a}(\Theta)(\lceil X\rceil)
\end{aligned}
$$




$$
\begin{aligned}
& \Leftrightarrow\left(\exists X^{\prime} \in \mathcal{F}_{\tau}, Y^{\prime} \in \mathcal{F}_{\tau^{\prime}}\right) X^{\prime} \sqsubseteq\lceil X\rceil \& Y \sqsubseteq\left\lceil Y^{\prime}\right\rceil \& X^{\prime} \Theta Y^{\prime} \\
& \Leftrightarrow\left(\exists Y^{\prime} \in \mathcal{F}_{\tau^{\prime}}\right) Y \sqsubseteq\left\lceil Y^{\prime}\right\rceil \& X \Theta Y^{\prime} \\
& \Leftrightarrow\left(\exists Y^{\prime} \in \mathcal{F}_{\tau^{\prime}}\right)\left(Y^{\prime},\lceil Y\rceil\right) \in \Theta_{\mathbb{Q}_{\tau^{\prime}}} \& X \Theta Y^{\prime} \\
& \Leftrightarrow(X,\lceil Y\rceil) \in \Theta_{\mathbb{Q}_{\tau^{\prime}}} \circ \Theta
\end{aligned}
$$

That is $\Theta_{\mathbb{Q}_{\tau^{\prime}}} \circ \Theta=\mathcal{H}_{a}\left(\mathcal{G}_{a}(\Theta)\right) \circ \Theta_{\mathbb{P}_{\tau}}$.

According to Propositions 14,15, 16, we obtain the following theorem.

Theorem 17 LDOM and LDFC are categorically equivalent.

\section{Declarations}

- Ethics approval

This article does not contain any studies with human participants or animals performed by any of the authors.

- Funding details This article is supported by National Natural Science Foundation of China(11771134).

- Conflict of interest

Both authors declare that they have no conflict of interest.

- Informed Consent Both authors agree to contribute this article to soft computing.

- Authors' contributions The two authors contributed half of the article.

\section{References}

[1] R.M. Amadio and P.L. Curien, Domains and Lambda-calculi, Cambridge University Press, Cambridge (1998)

[2] B. A. Davey and H. A. Priestley, Introduction to Lattices and Order, Cambridge University Press, Cambridge (2002)

[3] Ganter and R. Wille, Formal Concept Analasis, Springer-Verlag (1999)

[4] G. Gierz, K. H. Hofmann, K. Keimel, J. D. Lawson, M. Mislove, and D. S. Scott, Continuous Lattices and Domains, Cambridge University Press, Cambridge (2003)

[5] J. Goubault-Larrecq, Non-Hausdorff Topology and Domain Theory, Cambridge University Press, Cambridge (2013)

[6] L. Guo, F. Huang, Q. Li, and G. Zhang, Power contexts and their concept lattices, Discrete Mathematics, 311, 2049-2063 (2011) 
[7] L. Guo, Q. Li, and M. Huang, A categorical representation of algebraic domains based on variations of rough approximable concepts, International Journal of Approximate Reasoning, 55, 885-895 (2014)

[8] L. Guo, Q. Li, and L. Yao, Locally complete consistent F-augmented contexts: A category-theoretic representation of algebraic L-domains, Discrete Applied Mathematics, 249, 53-63 (2018)

[9] L.Guo, Q.Li, and G.Zhang, A representation of continuous domains via relationally approximable concepts in a generalized framework of formal concept analysis. International Journal of Approximate Reasoning, 114:2943, 2019.

[10] P. Hitzler, M. Kröetzsch, and G. Zhang, A categorical view on algebraic lattices in formal concept analysis, Fundamenta Informaticae, 74, 1-29 (2006)

[11] M. Huang, Q. Li, and L. Guo, Formal context for algebraic domains, Eletronic Notes in Theoretical Computer Science, 301, 79-90 (2014)

[12] A. Jung, Cartesian Closed Categories of Domains, CWI Tracts, Centrum voor Wiskunde en Informatica, Amesterdam, 66, (1989)

[13] A. Jung, The classification of continuous domains, 35-40, in: Proc, Fifth Annual IEEE Symposium on Logic in Computer Science, IEEE Computer Society Press, New York, (1990)

[14] H. Lai and D. Zhang, Concept lattices of fuzzy contexts: Formal concept analysis vs. rough set theory, International Journal of Approximate Reasoning, 50, 695-707 (2009)

[15] Y. Lei and M. Luo, Rough concept lattices and domains, Annals of Pure and Applied Logic, 159, 333-340 (2009)

[16] J. Li, C. Huang, J. Qi, Y. Qian, and W. Liu, Three-way cognitive concept learning via multi-granularity, Information Sciences, 378(1), 244-263 (2017)

[17] J. Li, C. Kumar, C. Mei, and X. Wang, Comparison of reduction in formal decision contexts, International Journal of Approximate Reasoning, 80, 100-122 (2017)

[18] Q. Li and L. Guo, Formal query systems on contexts and a representation of algebraic lattices, Information Sciences, 239, 72-84 (2013)

[19] S. Mac Lane, Categories for the Working Mathematician, volume 5 of Graduate Texts in Mathematics, Springer Verlag, (1971) 
[20] J. Poelmans, D. I. Ignatov, S. O. Kuznetsov, and G. Dedene, Formal concept analysis in knowledge processing: A survey on applications, Expert Systems with Applications, 40, 6538-6560 (2013)

[21] D. Scott, Outline of a mathematical theory of computation, In 4th Annual Princeton Conference on information Sciences and systemss, 169-176 (1970)

[22] D. Scott, Strachey C, Towards a mathematical semantics for computer languages, in: Proceeding of 21st Symposium on Computers and Automata. Polytechnic institute of Brooklyn, 19-46 (1971)

[23] D.Scott, Lattice theory, data type and semantics, Formal semantics of programming languages, 65-106, Prentice-Hall, Englewood Cliffs, N. J. (1972)

[24] Mislove M W, Topology, domain theory and Theoretical computer science, Topology and its Applications, 89(1,2): 3-59 (1998)

[25] L. Wang, L. Guo, Q. Li, Continuous Domains in Formal Concept Analysis. Fundamenta Informaticae, 179:295-319(2021)

[26] P. Valtchev, R. Missaoui, and P. Lebrun, A partition-based approach towards constructing Galois (concept) lattices, Discrete Mathematics, 256, 801-829 (2002)

[27] G. Zhang and G. Shen, Approximable concepts, Chu space, and information systems, Theory and Applications of Categories, 17(5), 80-102 (2006) 